Artículo

\title{
Descripción y análisis de productores de café de la región Mixe, Oaxaca
}

\author{
José Uriel García-Domínguez ${ }^{1}$ \\ Yuri Villegas-Aparicio ${ }^{1 \S}$ \\ Elvira Duran-Medina² \\ José Cruz Carrillo-Rodríguez ${ }^{1}$ \\ Dora Ma. Sangerman-Jarquín ${ }^{3}$ \\ Ernesto Castañeda-Hidalgo ${ }^{1}$
}

1Instituto Tecnológico del Valle de Oaxaca. Ex-Hacienda Nazareno, Santa Cruz Xoxocotlán, Oaxaca, México. CP. 71230. (urielgarcia.jugd@gmail.com; ernesto.ch@ voaxaca.tecnm.mx; jose.cr@voaxaca.tecnm.mx). ${ }^{2}$ Instituto Politécnico Nacional-CIIDIR Unidad Oaxaca. Hornos núm. 1003, Santa Cruz Xoxocotlán, Oaxaca, México. CP. 71230. (eduran3@hotmail.com). ${ }^{3}$ Campo Experimental Valle de México-INIFAP. Carretera los Reyes-Texcoco km 13.5, Coatlinchán, Texcoco, Estado de México, México. CP. 56250. (sangerman.dora@inifap.gob.mx).

${ }^{\S}$ Autor para correspondencia: yuriva1968@gmail.com.

\section{Resumen}

Diagnósticos sobre los integrantes de sectores productivos agrícolas proveen información para generar estrategias y políticas públicas que les fortalezcan, acordes a sus contextos. El objetivo del presente estudio fue describir y analizar a productores de café con base en aspectos sociales, productivos, económicos y su percepción sobre la cafeticultura. La información se obtuvo mediante entrevistas semiestructuradas realizadas entre noviembre de 2018 y marzo de 2019 a productores $(n=40)$ de la región Mixe, Oaxaca escogidos mediante un muestreo no probabilístico. La información se analizó con estadística descriptiva, análisis de conglomerados jerárquicos, prueba de Kruskal-Wallis, $\chi^{2}$ de Pearson y análisis de componentes principales. Los resultados mostraron que los productores desarrollan la cafeticultura con desventajas sociales, económicas y productivas donde la inversión en renovación de cafetal $\left(\chi^{2}=0\right)$, superficie en producción $\left(\chi^{2}=0\right)$, venta de café $\left(\chi^{2}=0\right)$, percepción sobre la cafeticultura como opción productiva $\left(\chi^{2}=0.001\right)$, edad de los productores $\left(\chi^{2}=0\right)$, años de experiencia $\left(\chi^{2}=0\right)$ y el grado de educación $\left(\chi^{2}=0\right)$ explican la conformación de productores inconformes (37.5\%), indecisos $(22.5 \%)$ y conformes (40\%). Los productores presentan carencias sociales, productivas y económicas que se manifiestan en una cafeticultura de subsistencia, agroecosistemas que tienden a disminuir su agrobiodiversidad, con estrategia parcial de plagas y enfermedades, y estructuras comerciales deficientes que orillan a una percepción sobre esta actividad productiva donde la mayoría está inconforme e indecisa.

Palabras clave: análisis de componentes principales, cafeticultores, organización.

Recibido: marzo de 2021

Aceptado: julio de 2021 


\section{Introducción}

Los agroecosistemas son fundamentales para la provisión de alimentos y productos claves para las sociedades contemporáneas, entre los más simbólicos se encuentran los agroecosistemas de café bajo sombra que representan los medios de vida de alrededor de 25 millones de pequeños productores en el mundo (Valencia et al., 2016; Wagner et al., 2019). El cultivo del café, como otros cultivos, opera en escenarios de incertidumbre debido a climas extremos, precios bajos, plagas y enfermedades, esta situación fue evidenciada con la reciente crisis de la roya (Hemileia vastatrix Berkeley y Broome) en Centroamérica y México. Esto, aunado a las características propias de los productores, hace que sean vulnerables al ubicarse en condiciones de marginación (Haggar et al., 2015; Läderach et al., 2017; Gerlicz, 2018; WCR, 2019).

Producir café en contextos sociales donde la escasez de recursos prevalece es un reto abordado con distintos enfoques como la innovación inclusiva (Amaro-Rosales y Gortari- Rabiela, 2016), el fortalecimiento organizacional (Espinoza et al., 2020), los medios de vida (Alonso et al., 2019) y la promoción del cooperativismo y el comercio justo (Arboleda et al., 2020; Ramírez y Nava, 2020). En México, la producción de café es una actividad que ocurre en 14 estados y cubre una extensión aproximada de 712000 ha. Actualmente, Oaxaca es el cuarto productor de café en el país con alrededor de 135000 ha cultivadas en regiones indígenas marginadas (Ruelas-Monjardín, 2014; Bara y Pérez, 2015; SAGARPA, 2016; Fernández y Méndez, 2018; Henderson, 2019; SIAP, 2019).

Una región poco estudiada es la zona Mixe ubicada al noreste del estado que cuenta con 15 municipios que practican esta actividad en un contexto de alta marginación y pobreza (CONEVAL, 2018). En varias regiones se desconocen las características de los productores aun cuando esto es un requisito previo para proponer estrategias que impulsen la agricultura (Borja et al., 2018). Es importante comprender contextos locales para generar estrategias y políticas públicas adecuadas, por esta razón, el presente trabajo tuvo como objetivo describir y analizar a los productores de café con bases en aspectos sociales, productivas, económicas y la percepción actual sobre la cafeticultura.

\section{Materiales y métodos}

\section{Área de estudio}

Este estudio descriptivo y analítico se realizó en cuatro localidades: Zompantle, San Isidro Huayapam, Santa Margarita Huitepec y Santiago Ixcuintepec (16 ${ }^{\circ} 56^{\prime} 05.7^{\prime} / 17^{\circ} 00^{\prime} 58.0^{\prime}$ ' latitud norte y $95^{\circ} 37^{\prime} 24.1^{\prime \prime} / 95^{\circ} 54^{\prime}$ 09.1" longitud oeste), ubicados en la región Mixe al noreste del estado de Oaxaca, México (Figura 1).

El área forma parte de la Sierra Madre del Sur y su topografía accidentada es propia de estas cadenas montañosas con pendientes pronunciadas (INEGI, 2016). Se encuentra inserta en una de las regiones terrestres prioritarias de México (RTP-130) que presenta diversidad de ambientes interconectados entre bosques mesófilos de montaña y selva alta perennifolia (Arriaga et al., 2000). Los habitantes de la zona pertenecen a la cultura Mixe y practican la agricultura de subsistencia, cafeticultura, ganadería extensiva, se encuentran en una categoría migratoria de fuerte expulsión y el producto de mayor comercialización en la región es el café (INPI, 2017). 


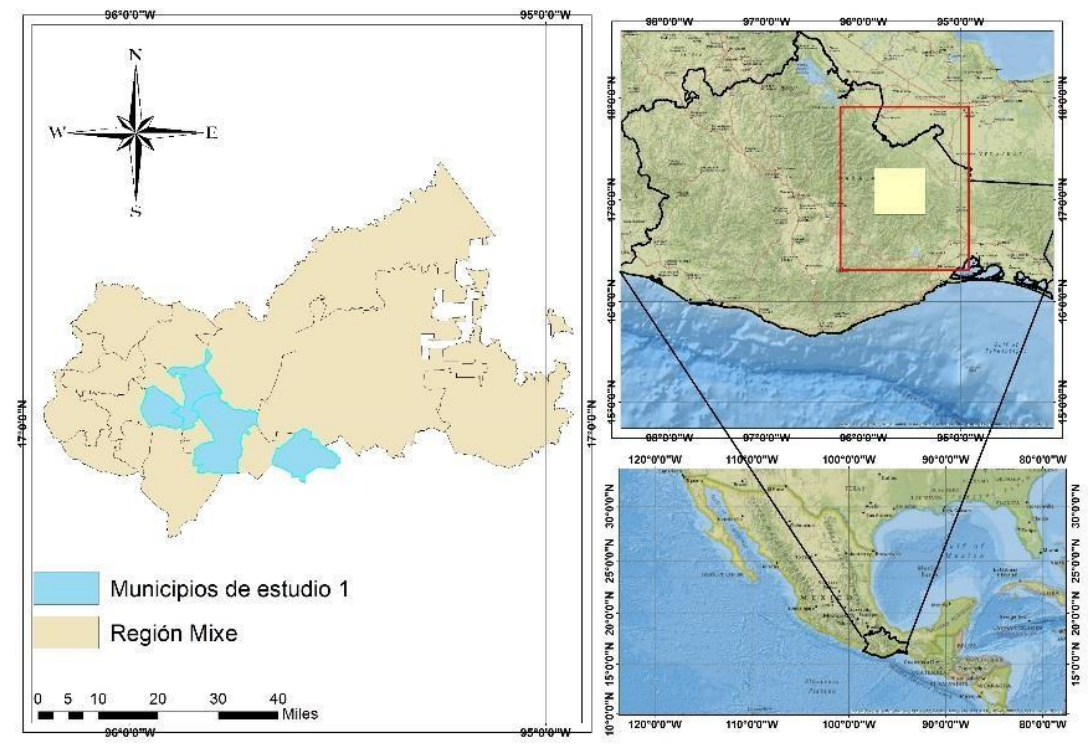

Figura 1. Área de estudio (INEGI, 2019).

Desde que el café cobró auge en la década de 1940 (Torres, 1997), la cafeticultura en esta región ha pasado por distintas etapas. Actualmente existen aproximadamente 5420 productores organizados en 16 agrupaciones y distribuidos en los cuatro municipios de estudio (AMECAFÉ, 2020). El estudio se realizó en tres etapas: 1) muestreo no probabilístico; 2) recolección de información; y 3) análisis estadístico de la información.

Muestreo probabilístico. Los datos de campo se recogieron mediante una técnica de muestreo en dos etapas. En primer lugar, se realizó un muestreo no probabilístico que condujo al caso de cuatro agrupaciones seleccionadas por disposición y accesibilidad de los sujetos, En segundo lugar, se escogieron aleatoriamente 40 productores para realizar el presente estudio de un total de 108 productores que integran las cuatro agrupaciones.

Recolección de información. Se realizaron entrevista semiestructurada de 25 preguntas organizadas en cuatro secciones: 1) aspectos sociales (género, edad del productor, número de integrantes en la familia, servicios básicos en la vivienda -agua, energía eléctrica, drenaje, combustible para cocinar-), escolaridad, servicio de asistencia médica); 2) aspectos productivos (historial de venta, años de experiencia, superficie total de parcela, superficie en producción, edad de las plantaciones (nuevos-viejos), manejo o labores culturales, tipo de cafetal -composición por variedades plantadas, especies para sombra y otras plantaciones útiles-); 3) aspectos económicos (ingreso familiar, programas sociales, fuentes de ingresos, canales de venta); y 4) percepción sobre la cafeticultura (como opción productiva y razón de respuesta) y de los problemática.

Se entrevistaron a 31 hombres y nueve mujeres $(n=40)$ escogidos al azar, éstas se realizaron en sus hogares y parcelas durante el periodo de noviembre de 2018 a marzo de 2019. Análisis estadístico de la información. La información reunida se concentró en una base de datos. Se hizo un análisis descriptivo y posteriormente se clasificaron a los sujetos de estudio en grupos mediante un análisis de conglomerados jerárquicos usando distancia euclídea al cuadrado y el método de Ward a fin de optimizar la homogeneidad de los clústeres. De los grupos formados, se analizaron las variables que influyen dicha conformación mediante pruebas de Kruskal-Wallis y $\chi^{2}$ de Pearson. Por último, 
las variables cuantitativas que mostraron significancia estadística $(p \leq 0.05)$ se sometieron a un análisis de componentes principales para explicar la conformación de los grupos. Los análisis se realizaron en el paquete estadístico SPSS V25.0 (2017).

\section{Resultados y discusión}

\section{Características sociales}

En términos organizativos, se destaca que los productores de café entrevistados están conformados por hombres $(68 \%)$ y mujeres (32\%) organizados en grupos locales de trabajo, esto refleja la tendencia de inclusión y relaciones de género en las estructuras de poder y los beneficios económicos (Cárcamo et al., 2010; Paz, 2018); sin embargo, las mujeres indígenas siguen viviendo en condiciones de exclusión y marginación (Muñoz y Vásquez, 2012). Respecto a los aspectos sociodemográficos, la edad promedio de los entrevistados es de 53.8 años, con familias de 3.6 integrantes en promedio y de acuerdo con su experiencia existen tres categorías: el $30 \%$ pertenecen a la generación de adultos mayores ( $\geq 61$ años), $57.5 \%$ a la generación productores maduros (36-60 años) y $12.5 \%$ a la generación de productores jóvenes ( $\leq 35$ años).

La disminución de productores jóvenes amenaza la continuidad de la cafeticultura por falta de relevo generacional, esto coincide con otros estudios (Aguirre et al., 2016; Jiménez-Barbosa et al., 2018) y en consecuencia, conlleva la pérdida de los beneficios sociales, económicos y ambientales que proveen estos sistemas reconocido en otros estudios como sostenibles (Haggar et al., 2017; Vanderhaegen et al., 2018; Ho et al., 2018; Morales et al., 2018).

Las viviendas de los entrevistados presentan carencia de al menos un servicio básico (100\% drenaje, $32.5 \%$ combustible para cocina y $32.5 \%$ agua entubada en casa), en cuanto a energía eléctrica todos cuentan con este servicio. Las políticas y programas gubernamentales de atención social y las que están enfocadas al sector cafetalero no han podido superar estas situaciones. Pocas iniciativas han logrado mejorar la calidad vida de los pequeños cafeticultores y se consideran casos de éxito (Pacheco et al., 2019); sin embargo, las condiciones de pobreza de los pequeños productores siguen reflejando los pocos beneficios que reciben.

El $80 \%$ se consideran analfabetas (primaria incompleta y sin educación formal), esto perjudica las iniciativas relacionadas con el desarrollo de capacidades que las políticas públicas o programas de comercio justo (Kudama, 2020) y en la lectura e interpretación de instrucciones de uso de insumos empaquetados que proveen programas sociales para el sector. Todos los productores reciben atención médica en las unidades médicas rurales de Instituto Mexicano del Seguro Social (UMRIMSS) y $57.5 \%$ recurre a médicos privados en casos de urgencia.

\section{Características productivas}

Con respecto a las condiciones productivas, el registro de venta de café pergamino seco refleja una pérdida que alcanzó 89\% en el ciclo 2011-2012 con respecto al ciclo anterior (Figura 2). Esta devastación de los cafetales a causa de la epidemia de roya impactó a nivel nacional y transformó las bases sociales, económicas y ecológicas de la cafeticultura en el largo plazo (Avelino et al., 2015; Renard y Larroa, 2017; Libert et al., 2019; Henderson, 2019). 


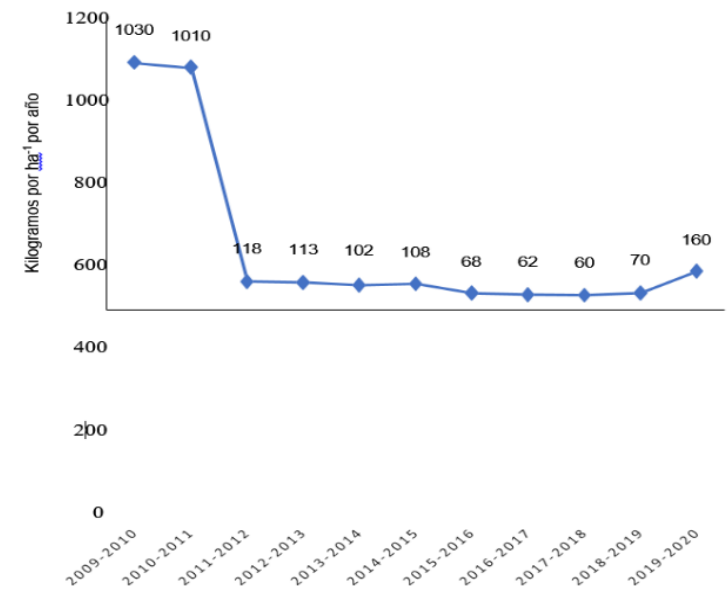

Figura 2. Historial de venta de café pergamino seco de cafeticultores encuestados $(n=40)$ en la región Mixe, Oaxaca, México.

Actualmente, los productores encuestados tienen en promedio 1.2 ha de cafetal que representa la mitad de la capacidad productiva. Los costos de renovación alcanzan los \$102000.00 ha-1 llevados a cabo en un periodo promedio de tres años, y respaldados por la práctica (Cuadro 1). Los cafetales se renovaron con trabajo propio, apoyo eventual de jornaleros y el auspicio de programas sociales para impulsar el sector (programa de apoyos a pequeños productores -componente PROCAFÉ-). De acuerdo con lo anterior, las características productivas de los encuestados corresponden a la categoría de pequeños productores, dado que poseen menos de cinco hectáreas de tierra, dependen principalmente de mano de obra familiar y solo contratan trabajadores de manera circunstancial (FAO, 2013; Bacon et al., 2014; Gómez et al., 2019).

Cuadro 1. Aspectos productivos actuales de cafeticultores encuestados $(n=40)$ en la región Mixe, Oaxaca, México.

\begin{tabular}{cccccc}
\hline Características & Media & DE & \multicolumn{2}{c}{ Mínimo } & Máximo CV (\%) \\
\hline Experiencia como cafeticultor (años) & 35.8 & 13 & 5 & 60 & 34.8 \\
Superficie total de la parcela (ha) & 2.3 & 1.3 & 1 & 5 & 55.8 \\
Superficie en producción (ha) & 1.2 & 0.6 & 0.25 & 3 & 53.1 \\
Edad de las plantaciones (años) & 10.5 & 7.54 & 3 & 18 & 71.81 \\
Costo de renovación $\left(\$ \mathrm{ha}^{-1} 3 \mathrm{años}^{-1}\right)$ & 102000 & 55510 & 45000 & 260000 & 49.08 \\
\hline
\end{tabular}

$\mathrm{DE}=$ desviación estándar; $\mathrm{CV}=$ coeficiente de variación.

En tanto al manejo de los cafetales, ésta ha sido predominantemente tradicional con esfuerzos de transición hacia la producción orgánica, en este sentido $85 \%$ de los productores implementan barreras vivas o muertas en curvas a nivel, cultivo de variedades resistentes a la roya, uso de insumos orgánicos para el aporte de nutrientes al suelo y posteriormente a los cafetales renovados. Todos los productores reconocen que este tipo de manejo implica más trabajo, también esperan un impacto económico positivo en el corto plazo como ya se ha constatado con otras experiencias (Calo y Wise, 2005; Rabanal et al., 2019; Rodríguez et al., 2019). 
Las áreas cafetaleras estudiadas se encuentran clasificados como policultivos tradicionales. En las áreas de estudio se distinguen dos subtipos: el 55\% se consideran altamente diversificados porque se encuentran entre 14 y 32 plantas útiles, hasta seis variedades de café y seis variedades de sombra, aunque actualmente tienen menor cantidad con respecto a lo reportado en otros estudios (Moguel y Toledo, 1999), los productores señalan que seguirán plantando más especies (Figura 3a) y 45\% presenta baja diversificación donde se encuentra principalmente una o dos variedades de café, y plátano e Inga vera sp., como sombra dominante con un proceso de diversificación lenta y los productores no dejan claro si seguirán esa tendencia (Figura 3b).

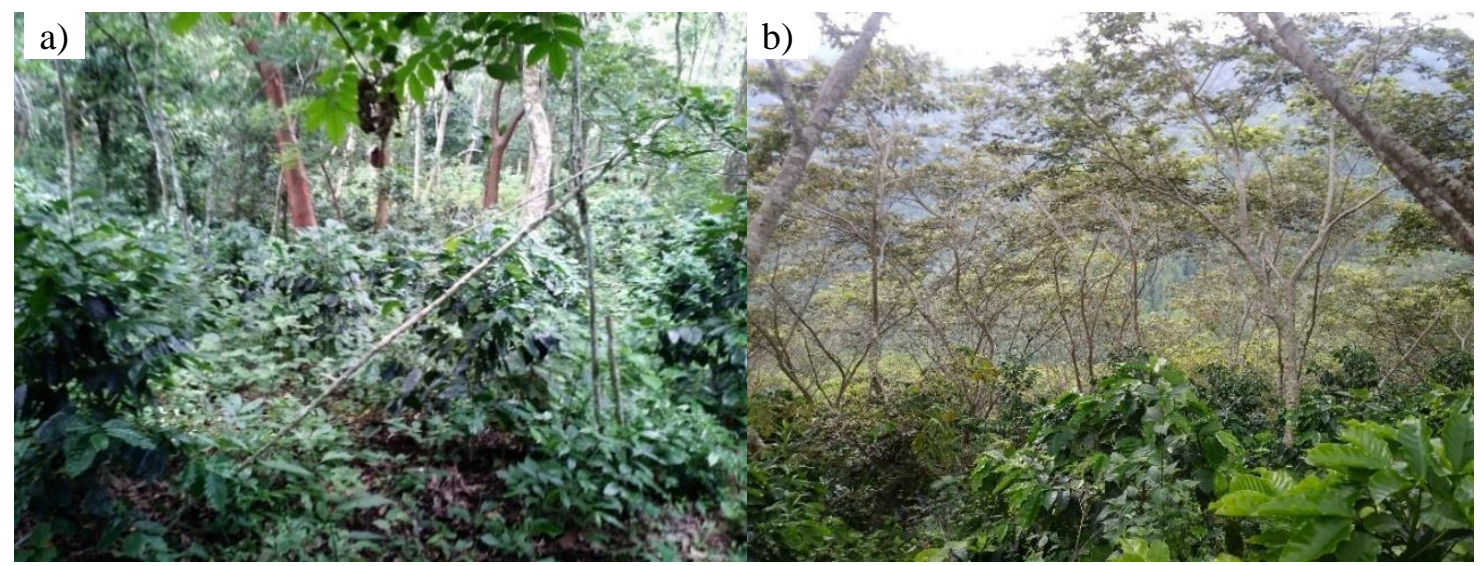

Figura 3a y 3b. Subtipos de cafetales con base en la agrobiodiversidad de pequeños productores $(n=$ 40) de la región Mixe, Oaxaca, México.

Los productores plantan más de una variedad de café de acuerdo con sus preferencias y facilidades de adquisición, hoy en día reportan que tienen las siguientes variedades de café: Marsellesa (97.5\%), Garnica (87.5\%), Costa Rica 95 (42.5\%), Mundo Novo (40\%), Caturra Amarrillo (30\%), Bourbon (17.5\%), Oro Azteca (15\%) y Criollo (7.5\%). Dentro de las parcelas, las variedades Marsellesa es la que predomina actualmente y cubre en promedio 67\% de los cafetales, 33\% restante corresponde a las otras variedades antes señaladas. Es importante recalcar que anteriormente la variedad predominante era el Criollo o Típica que fue devastada por la roya.

Las especies predominante de sombra son: Inga vera sp. (100\% -todos los productores la tienen plantada-), seguida de Musa paradisiaca L. (97.5\%), Persea americana (50\%), Citrus sinensis (47.5\%), Pouteria sapota (47.5\%), Cedrela odorata (32.5\%). La densidad promedio de Inga vera sp., es de 82 árboles por ha- ${ }^{-1}$, las otras variedades de sombra tienen una densidad irregular que no reportan los productores excepto la especie Cedrela odorata que en promedio se encuentran 6 árboles ha- ${ }^{-1}$.

\section{Características económicas}

Los productores tienen un ingreso promedio de \$2 432.00 pesos mensuales que provienen de la cafeticultura y de otros ingresos. El 5\% de los productores se apoya con la agricultura de autoconsumo, $62.5 \%$ con agricultura de autoconsumo y venta de mano de obra y $32.5 \%$ con agricultura de autoconsumo, remesas y/o programas sociales. La economía de los pequeños productores entrevistados obedece a las formas campesinas de diversificación de ingresos o a la multifuncionalidad de la agricultura familiar (Idárraga y Sánchez, 2016; Ticllacuri et al., 2020). 
Con respecto a los canales de venta, los productores pertenecen a una sociedad de producción rural de responsabilidad limitada (SPR de RL) donde se inscriben para comercializar el café producido y así enfrentar las situaciones de crisis cafetera, pero a pesar del esfuerzo, esta estructura ha tenido un desempeño limitado, en gran medida le atribuyen a la falta de manejo gerencial profesional e involucramiento de los socios, esto coincide con otros estudios (Rodríguez, 2020; Garduño y Ramírez, 2020).

\section{Percepción sobre la cafeticultura}

El 70\% de los productores considera que la cafeticultura es la mejor opción productiva en la región porque el cultivo está adaptado al entorno ecológico, social y cuenta con un mercado, esta percepción se ha ido construyendo socialmente en el marco de un contexto histórico-social, local y regional; a partir, de la experiencia y la posición de los productores en la cadena productiva que finalmente ha sido de subsistencia y reproducción social más que la acumulación de riquezas (Ejea, 2009; Rosales et al., 2018).

En el otro extremo está 30\% de los productores vacilantes y consideran la ganadería a pequeña escala, el cultivo mixto de amaranto y canela como mejores opciones locales y la migración para buscar mejores oportunidades de ingreso y calidad de vida (Figura 4). En tanto a la percepción de los problemas actuales, $67.5 \%$ considera que los precios bajos generan mayor aflicción para los productores, para $17.5 \%$ son las plagas y enfermedades y $15 \%$ considera que los climas extremos representan una amenaza.

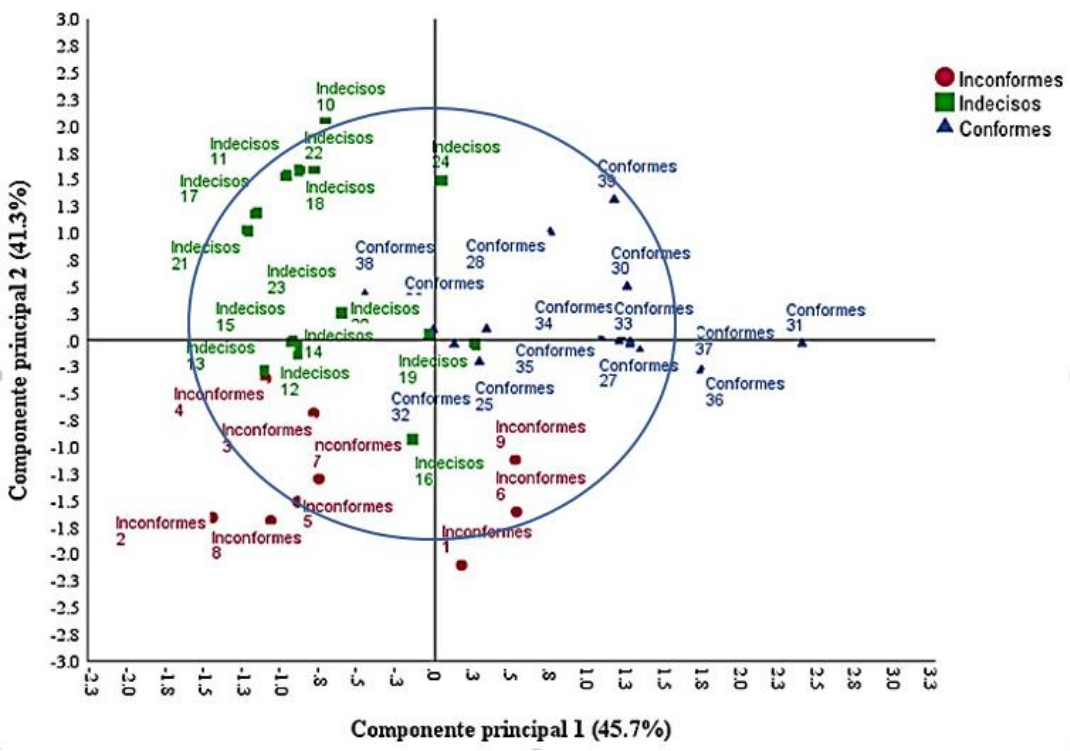

Figura 4. Dispersión de productores en función de los componentes principales.

\section{Diferenciación y análisis de los productores}

Se identificaron tres grupos mediante el análisis clúster a partir de las características sociales, económicas, productivas y de percepción social de la cafeticultura. El conglomerado I integró a $9(22.5 \%)$ productores, el conglomerado II agrupa a 15 productores (37.5\%) y el grupo III está conformado por 16 productores (40\%) (Figura 5). 

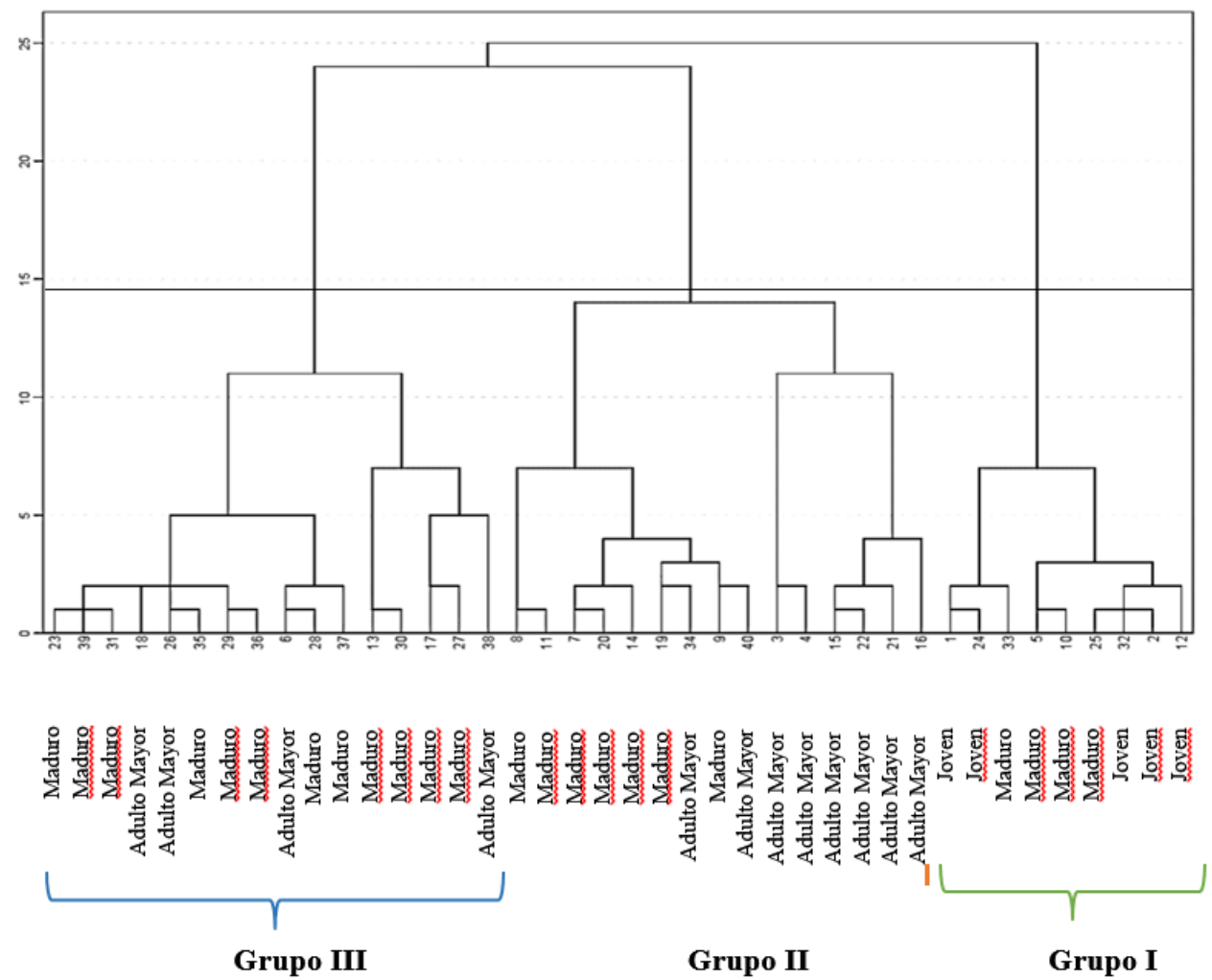

Figura 5. Agrupamiento de los productores mediante el método de Ward.

El grupo I está conformado por productores jóvenes que tienen en promedio 35.7 años, saben leer y escribir, lo que establece una diferencia significativa $\left(\chi^{2}=0\right)$ con respecto a los grupos II y III. Otro contraste, es que consideran la cafeticultura como una actividad poco atractiva $\left(\chi^{2}=0\right)$, esto se refleja en la renovación de sus cafetales, siendo de 0.9 ha en promedio, de los cuales, $66.7 \%$ presenta baja diversificación. Los productores del grupo II son lo que menos han invertido en la renovación de su cafetal ( $\$ 71733.30$ pesos) y cuentan con menor superficie promedio en producción (0.7 ha) de los cuales $86 \%$ de sus cafetales son altamente diversificados.

Una diferencia significativa $\left(\chi^{2}=0.001\right)$ es que tienen tres fuentes de ingreso porque han decidido explorar otras opciones como la ganadería o la migración temporal para venta de mano de obra. $\mathrm{Su}$ percepción sobre la cafeticultura es 'mala', marca diferencia significativa $\left(\chi^{2}=0.006\right)$ respecto a los otros grupos, esto es porque consideran que los precios bajos son el mayor problema. El grupo III está conformado por productores de edad avanzada, con mucha experiencia, fuerza laboral disminuida y se consideran analfabetas.

Son los productores que más han invertido en la renovación de sus cafetales, tienen mayor superficie en producción en hectáreas, de los cuales $75 \%$ son cafetales altamente diversificados y registraron mayor cantidad en ventas de café pergamino seco en el ciclo 2019-2020. Su percepción de la cafeticultura es muy buena, $93.8 \%$ considera que es un cultivo idóneo en términos económicos, sociales y ambientales (Cuadro 2). 
Cuadro 2. Valores (mínimo, máximo y media) y significancia de variables cuantitativas que conforman al grupo III.

\begin{tabular}{|c|c|c|c|c|}
\hline Variable & $\begin{array}{c}\text { Subgrupo I } \\
\quad(n=9)\end{array}$ & $\begin{array}{l}\text { Subgrupo II } \\
\quad(\mathrm{n}=15)\end{array}$ & $\begin{array}{l}\text { Subgrupo III } \\
\quad(\mathrm{n}=16)\end{array}$ & $\begin{array}{l}\text { Significancia } \\
\left(\chi^{2}=p<0.05\right)\end{array}$ \\
\hline Edad del productor(años) & $\begin{array}{c}35.3 \\
25 \pm 52\end{array}$ & $\begin{array}{c}61.8 \\
43 \pm 86\end{array}$ & $\begin{array}{c}56.8 \\
51 \pm 72\end{array}$ & 0 \\
\hline $\begin{array}{l}\text { Venta de café 2019- } 2020 \\
\qquad(\mathrm{~kg})\end{array}$ & $\begin{array}{c}117.2 \\
45 \pm 260\end{array}$ & $\begin{array}{c}94.4 \\
40 \pm 210\end{array}$ & $\begin{array}{c}247.8 \\
112 \pm 360\end{array}$ & 0 \\
\hline $\begin{array}{l}\text { Experiencia como cafeticultor } \\
\text { (años) }\end{array}$ & $\begin{array}{c}17.8 \\
5 \pm 35\end{array}$ & $\begin{array}{c}40.8 \\
20 \pm 60\end{array}$ & $\begin{array}{c}36.8 \\
25 \pm 55\end{array}$ & 0 \\
\hline $\begin{array}{l}\text { Superficie total de la parcela } \\
\text { (ha) }\end{array}$ & $\begin{array}{c}2.1 \\
1 \pm 4\end{array}$ & $\begin{array}{c}1.9 \\
1 \pm 4\end{array}$ & $\begin{array}{c}2.8 \\
1 \pm 5\end{array}$ & 0.154 \\
\hline Superficie en producción (ha) & $\begin{array}{c}0.9 \\
0.5 \pm 1.5\end{array}$ & $\begin{array}{c}0.7 \\
0.25 \pm 1.2\end{array}$ & $\begin{array}{c}1.8 \\
1 \pm 3\end{array}$ & 0 \\
\hline $\begin{array}{l}\text { Costo de renovación } \\
\left(\$ \mathrm{ha}^{-1} 3 \text { años }^{-1}\right)\end{array}$ & $\begin{array}{c}88111.1 \\
42000 \pm 145000\end{array}$ & $\begin{array}{c}71733.3 \\
30000 \pm 120000\end{array}$ & $\begin{array}{c}165937.5 \\
105000 \pm 260000\end{array}$ & 0 \\
\hline Ingreso familiar (\$) & $\begin{array}{c}2333 \\
1500 \pm 3600\end{array}$ & $\begin{array}{c}1980 \\
1500 \pm 3200\end{array}$ & $\begin{array}{c}2912 \\
1500 \pm 5000\end{array}$ & 0.059 \\
\hline
\end{tabular}

$\mathrm{El}$ análisis de componentes principales $(\mathrm{KMO}=0.721 p=0)$ generó dos componentes que explican $87.1 \%$ de la agrupación conformada (Figura 4). El componente 1 está definido por las variables de costo de renovación del cafetal, superficie en producción, venta de café pergamino seco, ciclo 2019-2020 y percepción sobre la cafeticultura, el componente 2 se define por la edad de los productores, los años de experiencia como cafeticultor y el nivel de educación.

Lo anterior explica y define los grupos de los productores inconformes con la cafeticultura porque consideran los bajos precios como el mayor problema (37.5\%), un grupo de productores indecisos en mantener esta actividad productiva $(22.5 \%)$ y los productores que están conformes con la cafeticultura $(40 \%)$.

\section{Conclusiones}

La situación social de los cafeticultores entrevistados no es favorable porque la mayoría de los productores son adultos mayores y no cuentan con relevo que asuma los retos que el sector implica en la actualidad. Se desempeñan con carencias de al menos un servicio básico en la vivienda, analfabetismo y servicio médico básico, esto deriva de problemáticas sociales a nivel de los municipios que son de bajo desarrollo humano. En términos productivos, los agroecosistemas de café son impactados por la roya y se han renovado lentamente, el manejo predominante sigue siendo tradicional con esfuerzos incipientes de transición hacia la producción orgánica, pero con una tendencia notable hacia la reducción que puede generar cambios importantes en la estructura y funcionamiento.

Existen productores inconformes con la cafeticultura porque consideran los bajos precios como el mayor problema, los indecisos en mantener estas actividades productivas y los que están conformes con la cafeticultura son adultos mayores. 


\section{Literatura citada}

Aguirre, C. J. F.; Cadena, I. J.; Ramírez, V. B.; Trejo, T. B. I.; Juárez, S. J. P. y Morales, F. F. J. 2016. Diversificación de cultivos en fincas cafetaleras como estrategia de desarrollo. Caso de Amatlán. Acta Universitaria. 26(1):30-38. https://doi.org/10.15174/au.2016.833.

Amaro-Rosales, M. y Gortari-Rabiela, R. 2016. Innovación inclusiva en el sector agrícola mexicano los productores de café en Veracruz. Economía Informa. (Ed.). 86-104 pp.

Arboleda, O. L.; Zavala, H. E. y Cueto, E. N. 2020. El cooperativismo caficultor en Colombia: el caso de la Cooperativa de Caficultores de Andes en el departamento de Antioquia, 19272015. América latina en la historia económica. 27(1):1-26. Doi: 10.18232/alhe.1025.

Arriaga, L. J. M.; Espinoza, C.; Aguilar, E.; Martínez, L.; Gómez y Loa, E. 2000. Regiones terrestres prioritarias de México. Escala de trabajo. 1:1 000 000. Comisión Nacional para el Conocimiento y uso de la Biodiversidad. México. 611 p.

AMECAFÉ. 2020. Asociación mexicana del café. Productores y productoras (información a nivel municipal): Total de productores del estado de Oaxaca, desglosado por municipio. México. http://www.cafeybiodiversidad.mx/productores-mun.php?label=oaxaca\&datos=1,109940 \&id-estado $=20$ \&persona $=$.

Avelino, J.; Cristancho, M.; Georgiou, S.; Imbach, P.; Aguilar, L.; Borne-mann, P. L.; Anzueto, F. y Hruska, C. M. 2015. The coffee rust crises in Colombia and Central America (20082013): impacts, plausible causes and proposed solutions. Food Securit. 7(2):303-321. doi: 10.1007/s12571-015-0446-9.

Bacon, C. M.; Sundstrom, W. A.; Flores, G. M. E.; Méndez, V. E.; Santos, R.; Goldoftas, B. y Dougherty, I. 2014. Explaining the 'hungry farmer paradox': smallholders and fair-trade cooperatives navigate seasonality and change in nicaragua's corn and coffee markets. Global Environmental Change. 25:133-149. doi: 10.1016/j.gloenvcha.2014.02.005.

Bara, C. y Pérez, P. 2015. Status quo, desafíos y oportunidades del café alternativo que se produce en México y se consume en Alemania. Agric. Soc. Des. 12(1):59-86.

Borja, B. M.; Velez, I. A. y Ramos, G. J. L. 2018. Tipología y diferenciación de productores de guayaba (Psidium guajava L.) en Calvillo, Aguascalientes, México. Región y Sociedad. 71(30):3-22. https://doi.org/10.22198/rys.2018.71.a402.

Calo, B. M. and Wise, T. A. 2005. Revaluing peasant coffee production: organic and fair-trade markets in Mexico, Medford, Mass. Global Development and Environment Innstitute, October. http://ase.tufts.edu/gdae/pubs/rp/RevaluingCoffee05.pdf.

Cárcamo, T. N. J.; Vázquez, G. V.; Zapata, M. E. y Nazar, B. A. 2010. Género, trabajo y organización. Mujeres cafetaleras de la unión de productores orgánicos de San Isidro Chiltepec, Chiapas. Estudios Sociales. 18(36):156-176.

CONEVAL. 2018. Consejo nacional de evaluación de la política de desarrollo social. Informe de pobreza y evaluación 2018. Oaxaca. Ciudad de México. 2018. https://www.coneval.org.mx /coordinacion/entidades/documents/informes-de-pobreza-y-evaluacion2018Documentos/ InformeOaxaca2018.pdf.

Ejea, M. T. 2009. Café y cultura productiva en una región de Veracruz. Nueva Antropología. 70 (22): 33-56. <http://www.scielo. org.mx/scielo.php?script=sci_arttext\&pid=S0185-063620 $09000100003 \& \operatorname{lng}=$ es\&nrm=iso $>$. ISSN 0185-0636.

Espinoza, L. V.; Rojas, D.; Salas, T. E. E. y Samaniego, N. A. 2020. Fortalecimiento organizacional de asociaciones de productores de café en las provincias de Loja y Zamora Chinchipe, Ecuador. Vínculos-Espe. 5(1):25-36. 
Fernandez, M. y Méndez V. E. 2018. Subsistence under the canopy: agrobiodiversity's contributions to food and nutrition security amongst coffee communities in Chiapas, Mexico. Agroecol. Sustain. Food Syst. 1-23 pp. doi:10.1080/21683565.2018. 1530326.

FAO. 2013. Food and agriculture organization of the United Nations. Agricultores pequeños y familiares. Vías de sostenibilidad. http://www.fao.org/3/ar588s/ar588s.pdf.

Garduño, Á. G. y Ramírez, L. A. 2020. Beneficios de pertenecer a una organización cafetalera: el caso de Santa María Yucuhiti, Oaxaca. Problemas del desarrollo Rev. Latinoam. Econ. 5(202):91-116. https:// doi.org /10 .22 201 /iiec.20078951e.2020.202.69516.

Gerlicz, A.; Méndez, V. E.; Conner, D.; Baker, D. and Christel, D. 2018. Use and perceptions of alternative economic activities among smallholder coffee farmers in Huehuetenango and el Quiché departments in Guatemala. Agroecol. Sustain. Food Syst. 1-19 pp. doi:10.1080/21683565.2018.1532480.

Gómez, M. E.; Barradas, P. y Sámano-Rentería, M. A. 2019. Condiciones sociales que caracterizan la multifuncionalidad de la agricultura en México. Campo território: Rev. Geogr. Agrár. 14(32):7-27. https://doi.org/10.14393/RCT143201.

Haggar, J.; Asigbaase, M.; Bonilla, G.; Pico, J. and Quilo, A. 2015. Tree diversity on sustainably certified and conventional coffee farms in Central America. Biod. Conserv. 24(5):11751194. doi: 10.1007/s10531-014-0851- y.

Haggar, J.; Soto, G.; Casanoves, F. and Virginio, E. de M. 2017. Environmental-economic benefits and trade-offs on sustainably certified coffee farms. Ecol. Indicators. 79:330-337. doi: 10.1016/j.ecolind.2017.04.023.

Henderson, T. P. 2019. La roya y el futuro del café en Chiapas. Rev. Mex. Sociol. 81(2):389-416. Ho, T. Q.; Hoang, V. N.; Wilson, C. y Nguyen, T. T. 2018. Eco-efficiency analysis of sustainability-certified coffee production in vietnam. J. Cleaner Production. 183:251-260. doi: 10.1016/j.jclepro.2018.02.147.

Idárraga, Q. Á. y Sánchez R. J. J. 2016. Agricultura familiar ambientalmente sustentable y económicamente sostenible: estudio de caso de la asociación de pequeños productores de café (Asopecam). Estudios de caso desde la multifuncionalidad y su aporte a la paz Bogotá: (Ed.). Universidad Cooperativa de Colombia. 97-120 pp.

INEGI. 2016. Instituto Nacional de Estadística y Geografía. Anuario estadístico y geográfico de Oaxaca 2016. Instituto Nacional de Estadística y Geografía. México. 2016. http://internet.contenidos.inegi.org.mx/contenidos/Productos/prod-serv/contenidos/ español/bvinegi/productos/nueva-estruc/AEGPEF-2016/702825087357.pdf.

INPI. 2017. Instituto Nacional de los Pueblos Indígenas Etnografía del pueblo Mixe de Oaxaca (ayuukjä'äy). Blog del Instituto Nacional de los Pueblos Indígenas. https://www.gob.mx/ inpi/articulos/etnografia-del-pueblo-mixe-ayuukja-ay\#: :text=Localizaci\%C3\%B3n,total $\% 20 \mathrm{de} \% 204 \% 20668.55 \% 20 \mathrm{~km} 2$.

Jiménez-Barbosa, W. G.; De la Portilla E.; Zúñiga L. A.; Zambrano D. F.; Rojas J. S. y Delgado, R. A. 2018. Relevo generacional para la continuidad de producción cafetera familiar. Caso municipio de Albán, Nariño-Colombia. Rev. Colomb. Cienc. Soc. 10(1):67-92 doi: https://doi.org/10.21501/22161201.3060.

Kudama, G. 2020. Determinants of fairtrade channel choice and its effect on income of coffee farm households. research square. doi: 10.21203/rs.3.rs-21332/v1.

Läderach, P.; Ramírez-Villegas, J.; Navarro-Racimes, C.; Zelaya, C.; Martínez-Valle, A. and Jarvis, A. 2017. Climate change adaptation of coffee production in space and time. Climatic change 141(1):47-62. Doi: 10.1007/s10584-016-1788-9. 
Libert, A. A. y Paz-Pellat, F. 2019. Del papel a la acción en la mitigación y adaptación al cambio climático: la roya del cafeto en Chiapas. Madera y Bosques. 24(esp.):1-24. 2401914. https://dx.doi.org/10.21829/myb.2018.2401914.

Moguel, P. y Toledo, V. M. 1999. Biodiversity conservation in traditional coffee systems of Mexico. Conserv. Biol. 13(1):11-21. doi:10.1046/j.1523- 1739.1999. 97153. X.

Morales, H. J. I.; Méndez, M. L.; Nolasco R. S. P. and Cerón L. M T. 2018. Proposal for the creation of a network of family businesses in the mexican coffee industry. Inter. J. Adv. Eng. Manag. Sci. (IJAEMS). 4(11):773-781. https://dx.doi.org/10.22161/ijaems.4.11.6.

Muñoz, R. C. y Vázquez, G. V. 2012. El Estado neoliberal y las mujeres indígenas: Un estudio de caso de la sierra negra de puebla. Espiral. 19(53):91-121.

Pacheco, A. P.; Palacios, R. M. I.; Cervantes, E. F.; Ocampo, L. J. y Aguilar, Á. J. 2019. La asociación cooperativa como factor de sostenibilidad del sistema cafetalero en comunidades marginadas. Rev. Estudios Cooperativos. 131:125-150. http://dx.doi.org/ 10.5209/REVE.63563.

Paz, P. L. 2018. Mujeres del cafetal en la región central de Veracruz. Aportaciones femeninas a la economía campesina en el colapso de 2013-2017. Argumentos, estudios críticos de la sociedad. 31(86):229-250.

Rabanal Á. J.; Pinglo J. F. M.; Vásquez M. E. E.; Cayo-Colca I. S. y Guivin, G. L. 2019. Análisis del impacto económico de certificación orgánica de café (Coffea arabica) en la cooperativa agraria Alta Montaña- COOPAM- 2018. Rev. Investigación Científica UNTRM. Ciencias Naturales e Ingeniería. 1(2):44-47.

Ramírez G. S. y Nava, T. M. E. 2020. Comercio justo y empoderamiento de pequeños cafeticultores del centro de Veracruz. Agric. Soc. Des. 4(16):477-495.

Renard, H. M. C. y Larroa, T. R. M. 2017. Política pública y sustentabilidad de los territorios cafetaleros en tiempos de roya: Chiapas y Veracruz. Estudios Latinoam. Nueva Época. 40:95-113.

Rodríguez, H. D. M. 2020. Asociacionismo y cambio social en comunidades rurales andinas. Aproximación al caso de los cafeteros colombianos. Rev. Del CESLA. Inter. Latín Am. Studies Review. 25:57-79. https://doi.org/10.36551/2081-1160.2020.25.57-79.

Rodríguez, T. L. E.; Cárcamo, M. R. W. y Álvarez, M. A. 2019. La opción de la producción orgánica ante la crisis del café: el caso de los pequeños productores de Chilón, Chiapas, México. Sociedades Rurales, Producción y Medio Ambiente. 19(37):45-72.

Rosales, M. V.; Martínez, D. J. P.; Osorio, A. F.; López, R. G.; Asiaín, H. A. y Estrella, Ch. N. 2018. Aspectos culturales, sociales y productivos para una tipología de cafeticultores. Agric. Soc. Des. 15(1):47-61.

Ruelas-Monjardín, L. C.; Nava-Tablada, M. E.; Cervantes, J. y Barradas, V. L. 2014. Importancia ambiental de los agroecosistemas cafetaleros bajo sombra en la zona central montañosa del estado de Veracruz, México. Madera y Bosques. 20(3):27- 40.

SAGARPA. 2016. Secretaría de Agricultura, Ganadería, Desarrollo Rural, Pesca y Alimentación. Planeación agrícola nacional 2017-2030. Café mexicano. Ciudad de México, 2017. https://www.gob.mx/cms/uploads/attachment/file/256426/b-sico-caf-.pdf.

SIAP. 2019. Sistema de Información Agroalimentaria y Pesquera. Panorama agroalimentario 2019. Sistema de Información Agroalimentaria y Pesquera- Secretaría de Agricultura y Desarrollo Rural. Ciudad de México. 2019. https://nube.siap.gob.mx/gobmxpublicaciones-siap/pag/2019/atlas- agroalimentario-2019. 
Ticllacuri, M. A. y Wesz, J. V. J. 2020. Análisis económico del cultivo de café en los caseríos de alto Yacusisa y alto Belén (José Crespo y Castillo-Huánuco-Perú). Agronegocios. 6(2):121-136. https://doi.org/10.18845/ea.v6i2.4993.

Valencia, V.; Naeem, S.; García-Barrios, L.; West, P. and Sterling, E. J. 2016. Conservation of tree species of late succession and conservation concern in coffee agroforestry systems. Agr. Ecosyst Environ. 219:32-41.

Vanderhaegen, K.; Akoyi, K. T.; Dekoninck, W.; Jocqué, R.; Muys, B.; Verbist, B. and Maertens, M. 2018. Do private coffee standards 'walk the talk' in improving socio-economic and environmental sustainability? global environmental change. 51:1-9. doi: 10.1016/j. gloenvcha. 2018.04.014.

Wagner, S.; Rigal, C.; Liebig, T.; Mremi, R.; Hemp, A.; Jones, M. and Preziosi, R. 2019. Ecosystem services and importance of common tree species in coffee agroforestry systems: Local knowledge of small-scale farmers at Mt. Kilimanjaro, Tanzania. Forests. 10(11):963. doi: 10.3390/f10110963. 